\title{
Factores personales que inciden en la autovaloración de futuros maestros sobre la dimensión pedagógica del uso de TIC
}

\author{
Carolina Flores-Lueg y Rosabel Roig-Vila
}

\section{RESUMEN}

Se presentan los principales hallazgos de un estudio que se centró en determinar aquellos factores que tienen incidencia en la valoración de estudiantes de pedagogía sobre su nivel de competencia para el uso pedagógico de las tecnologías de la información y la comunicación (TIC). Se consideraron variables personales (edad, sexo, capacitación en TIC, nivel educativo de formación inicial y conectividad) y su incidencia en los niveles de competencia digital autopercibido por 175 estudiantes pertenecientes a las carreras de Pedagogía de una universidad chilena de carácter público. Se empleó una escala diseñada ad hoc para el estudio, la que obtuvo muy buenas cualidades psicométricas en el proceso de validación. Los resultados arrojan que los futuros maestros sienten poseer ciertos conocimientos sobre el uso de TIC desde una perspectiva pedagógica, pero en el marco de un enfoque tradicional. Las variables personales que tienen incidencia en dicha valoración fueron la edad, el sexo, el nivel educativo de formación inicial docente.

Palabras clave: TIC, formación de profesores, educación superior, competencias digitales, autopercepción, Chile.

Carolina Flores-Lueg

cflores@ubiobio.cl Chilena. Doctora en Investigación Educativa, Universidad de Alicante, España; Magíster en Ciencias de la Educación, Universidad Católica de la Santísima Concepción, Chile; Máster en Educación y Tecnologías de la Información y Comunicación aplicadas a educación, Universidad de Alicante, España. Docente del Departamento de Ciencias de la Educación, Facultad de Educación y Humanidades de la Universidad del BíoBío, Chile. Temas de investigación: educación y TIC; formación del profesorado.

Rosabel Roig-Vila

Española. Doctora en Pedagogía por la Universidad de Alicante, España. Profesora Titular del Área de Didáctica y Organización Escolar en el Departamento de Didáctica General y Didácticas Específicas, Directora del Instituto de Ciencias de la Educación y Directora del grupo de investigación EDUTIC-ADEI (Educación y TIC. Atención a la Diversidad. Escuela Inclusiva) (VIGROB-039), Universidad de Alicante, España. Temas de investigación: tecnologías de la información y la comunicación aplicadas a la educación. 


\title{
Fatores pessoais que incidem na autoavaliação de futuros professores sobre a dimensão pedagógica do uso de TIC
}

\section{RESUMO}

Apresentam-se as principais descobertas de um estudo que se centrou em determinar aqueles fatores que têm incidência na avaliação de estudantes de Pedagogia sobre seu nível de competência para o uso pedagógico de técnicas de informação e comunicação (TIC). Consideraram-se variáveis pessoais (idade, sexo, capacitação em TIC, nível educativo de formação inicial e conectividade) e sua incidência nos níveis de competência digital percebido por 175 estudantes do curso de Pedagogia de una universidade chilena de caráter público. Empregou-se uma escala desenhada ad hoc para o estudo, a que obteve muito boas qualidades psicométricas no processo de validação. Os resultados demonstram que os futuros professores sentem possuir certos conhecimentos sobre o uso de TIC por uma perspectiva pedagógica, mas no marco de um enfoque tradicional. As variáveis pessoais que tem incidência em dita avaliação foram a idade, o sexo, o nível educativo de formação inicial docente.

Palavras chave: tecnologias da informação e a comunicação, formação de professores, educação superior, competências digitais, autopercepção, Chile.

\section{Personal factors influencing future teachers' self-assessment about the pedagogical dimension of ICT use}

\begin{abstract}
This article presents the main findings of a study that focused on determining the factors that have an impact on the assessment of students of Pedagogy about their level of competence for the pedagogical use of information and communication techniques (ICT). Personal variables were considered (age, sex, ICT training, educational level of initial training and connectivity) and their impact on the levels of self-perceived digital competence by 175 students belonging to the Pedagogy degree of a Chilean public university. An ad hoc scale was used for the study that achieved very good psychometric qualities in the validation process. The results show that future teachers feel they have some knowledge about the use of ICT from a pedagogical perspective, but within the framework of a traditional approach. The personal variables that had an impact on this assessment were age, sex, and the level of initial teacher training.
\end{abstract}

Key words: information and communication technologies, teacher training, higher education, digital skills, self-perception, Chile.

Recepción: 25/04/17. Aprobación: 5/03/18. 


\section{Introducción}

Dentro del actual escenario mediado por las tecnologías de la información y la comunicación (TIC), sin duda han ido emergiendo una serie de desafíos que deben sortear los ciudadanos para poder acceder a la información que circula en internet y seleccionar aquella requerida; para llevar a cabo una serie de actividades de carácter cotidiano; para participar dentro de los distintos escenarios comunicativos que se han ido configurando e interactuar activamente en los espacios sociales y culturales, entre diversas otras demandas. En concreto, hoy en día las transformaciones generadas en distintos ámbitos sociales, como consecuencia de la marcada presencia de las TIC, han conducido al requerimiento de un nuevo tipo de alfabetización, la alfabetización tecnológica o digital. En este sentido, es importante tener en cuenta que el dinamismo con el que avanzan y se desarrollan estas herramientas le exige a una persona no sólo estar alfabetizada digitalmente, sino que al mismo tiempo le demanda la adquisición de otro tipo de alfabetizaciones, como por ejemplo, la alfabetización informacional, la alfabetización múltiple, entre otras. Por otro lado, es importante subrayar que el acceso a la información disponible en la Red y las posibilidades de participación activa en ambientes virtuales, exige el ejercicio de determinados derechos y deberes, por lo que además una persona debe moverse dentro del marco de la denominada ciudadanía digital.

En consecuencia, se puede decir que la educación claramente adopta una relevancia sin precedentes, pues en ella recae la responsabilidad de formar a los ciudadanos para el siglo XXI, quienes no sólo deben ser capaces de manifestar apertura frente a las nuevas posibilidades que estas herramientas ofrecen/ofrecerán o estar alfabetizados para hacer uso de los nuevos medios tecnológicos/digitales que irán emergiendo, sino que, además, requieren desarrollar ciertas competencias que les permitan aprender durante toda la vida (lifelong learning) y que los prepare para participar activamente dentro de este mundo cada vez más globalizado. Por tanto, más que alfabetizar digitalmente, en la actualidad se requiere promover el desarrollo de la competencia digital en sus diversas dimensiones.

Ahora bien, si se tiene en consideración que la acción educativa es eminentemente una acción social, situada en un tiempo y en un espacio determinado, el proceso formativo de cualquier persona no puede ser comprendido al margen de las características contextuales donde se lleva a cabo, y si las TIC han generado cambios de gran envergadura a nivel de la sociedad, la escuela y el profesorados se encuentran con el desafío de afrontar estos cambios e incorporar dichas herramientas no sólo con el fin de optimizar sus procesos administrativos sino que principalmente asumirlas como parte de los procesos educativos. Sin embargo, para que esta condición se logre, un profesor no sólo debe desarrollar la competencia digital como cualquier ciudadano, sino que además se requiere que desarrolle la competencia digital docente, como condición indispensable para el aprovechamiento de todo el aporte educativo que ofrecen actualmente las tecnologías, fundamentalmente las digitales, pero bajo una concepción epistemológica de la práctica docente renovada, más pertinente a las necesidades educativas actuales, pues concordamos con Vásquez-Cuperio y López-Penedo (2016: 251), cuando advierten que "los sistemas educativos están tratando de afrontar la epísteme del siglo XXI con un marco epistemológico del siglo XIX". Por consiguiente, el reto principal que se debe asumir desde la educación no está en transformar los procesos formativos a partir de la innovación tecnológica, sino con base en la innovación pedagógica apoyada por herramientas tecnológicas, desafío que debe ser abordado desde la formación inicial docente.

Teniendo en consideración lo anteriormente señalado, este artículo presenta los principales resultados obtenidos en una investigación que tuvo 
como propósito llegar a determinar aquellos factores que inciden en la valoración de estudiantes de Pedagogía sobre su nivel de competencia en el uso pedagógico de las TIC. En un primer apartado se presentan antecedentes teóricos que dan cuenta de las distintas dimensiones que componen la competencia digital que debe lograr desarrollar cualquier ciudadano en el escenario actual, posteriormente se hace referencia a aquellos aspectos específicos vinculados a la competencia digital que debe lograr desarrollar el profesorado, considerando para ello la dimensión aspectos pedagógicos definida en los estándares TIC para la Formación Inicial Docente en el contexto chileno, dada su plena pertinencia al escenario y nivel educativo considerado para llevar a cabo el estudio. En un segundo apartado se presentan los objetivos específicos definidos y se indica detalladamente la forma que se llevó a cabo el proceso investigativo desarrollado. En la tercera sección se exhiben primeramente los principales resultados descriptivos obtenidos a partir de la medición de cada una de las variables consideradas, los que permiten apreciar el nivel de competencia autopercibido por los futuros maestros en el uso pedagógico de las TIC. Posteriormente se presentan los principales resultados evidenciados al establecer el cruce entre determinadas variables, y cuyos niveles de significación/no significación se constituyeron en aquellos factores que inciden/no inciden en el nivel de competencia en el uso pedagógico de TIC autopercibido por los futuros maestros. Finalmente se discuten los principales resultados a la luz de los antecedentes teóricos que le dieron sustento al estudio, advirtiendo al mismo tiempo sobre algunos aspectos que se deberían tener en consideración para contribuir al mejoramiento de los procesos formativos vinculados al uso pedagógico de las TIC, por parte de los futuros egresados de las carreras de Pedagogía, particularmente de una universidad de carácter público y estatal, ubicada en Chile.

\section{Fundamentos teóricos: competencia digital docente}

Hacer alusión a la competencia digital no es una cuestión fácil, pues en la literatura se pueden encontrar una serie de definiciones que de una u otra forma buscan otorgarle un significado (Comisión de las Comunidades Europeas, 2005; Gisbert et al., 2011; Larrazt, 2012; Gutiérrez, 2014), estas definiciones integran aspectos que van desde elementos referidos a la alfabetización informacional hasta la consideración de aspectos éticos y legales. Sumado a lo anterior, diversos autores (Adell, 2010; Fainholc et al., 2013; Ferrari, 2013), hacen alusión a determinadas dimensiones de la competencia digital, destacándose algunas coincidencias entre las propuestas, principalmente en lo referido a la dimensión informacional, tecnológica y comunicativa. En consecuencia, se asume que la competencia digital es un tipo de competencia multidimensional (ver figura 1) y puede ser definida como la capacidad para movilizar aquellas habilidades y destrezas que permiten buscar, seleccionar críticamente, obtener y procesar información relevante haciendo uso de las TIC para transformarla en conocimiento, al mismo tiempo que se es capaz de comunicar dicha información a través de la utilización de diferentes soportes tecnológicos y digitales, actuando con responsabilidad, respetando las normas socialmente establecidas y aprovechando estas herramientas para informarse, aprender, resolver problemas y comunicarse en distintos escenarios de interacción (Flores, 2014).

Para algunos autores (Adell, 2010; Area, 2008; Larrazt, 2012), la dimensión Alfabetización informacional está referida a la capacidad para gestionar la información, aludiendo a un proceso que comprende acciones tales como: definir la información que se desea obtener o reconocer la necesidad de información; buscar o localizar la información en bases de datos o archivos de cualquier naturaleza; evaluar los resultados de búsqueda; organizar la información; 


\section{Figura 1. Dimensiones de la competencia digital.}

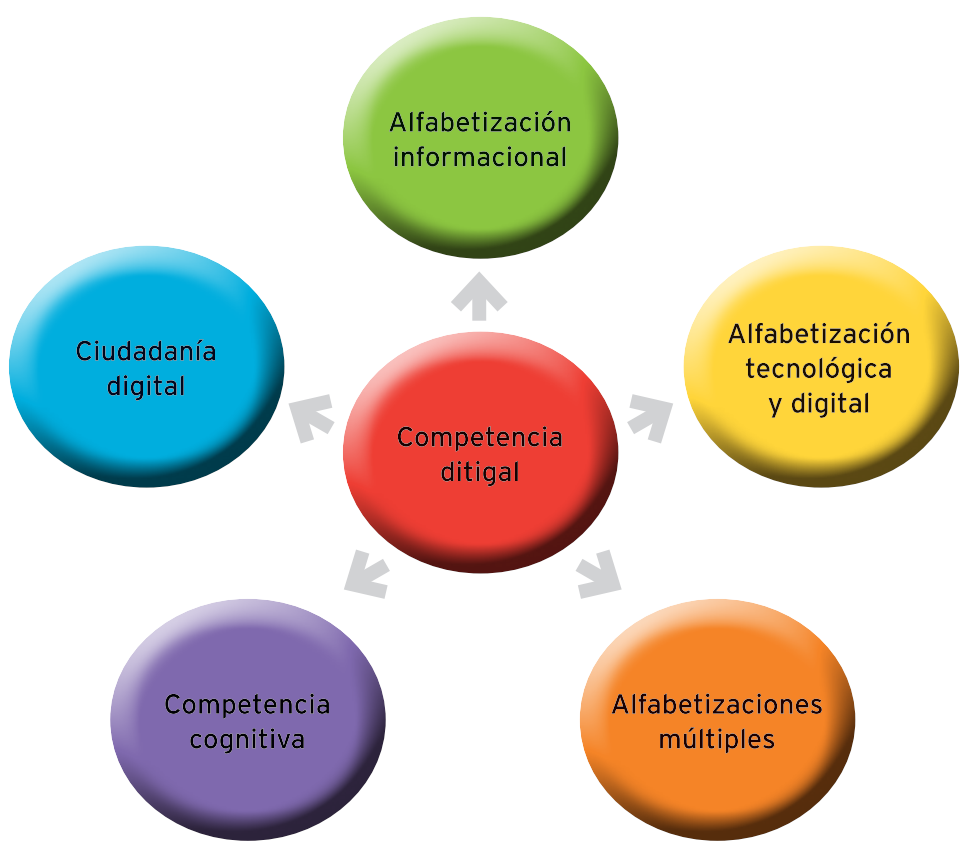

Fuente: adaptado de Adell, (2010), "Las 5 dimensiones de la competencia digital", parte Il de la ponencia "La Competencia Digital", XXIV Jornadas Pedagógicas de Barakaldo.

crear nueva información o conocimiento y comunicarla. En este sentido, la alfabetización informacional implica saber plantear estrategias y procesos de resolución de problemas relacionados fundamentalmente con la información (Area, 2008).

La dimensión Alfabetización tecnológica y digital está relacionada con el manejo de la tecnología digital para la búsqueda, selección y organización de la información, además de la capacidad para compartirla en los diferentes formatos disponibles, principalmente los multimedia, así como también para interactuar en los distintos entornos comunicativos que estas tecnologías están conformando (Flores, 2015).

En cuanto a la dimensión Alfabetizaciones múltiples, se concuerda con Adell (2008), cuando señala que ésta está referida a la capacidad para ser espectador crítico y creador de mensajes audiovisuales, icónicos, entre otros; es decir, ser capaz de movilizar y utilizar las nuevas herramientas de información y comunicación disponibles, para ello "necesitan estar alfabetizados no sólo en los medios impresos y sus códigos verbales, sino que también en los recursos multimedia" (Cabero, Marín y Llorente, 2012: 11).

La dimensión Alfabetización cognitiva refiere al aprender a utilizar de forma inteligente la información disponible a través de las distintas tecnologías, "tanto para acceder a la misma como para recrearla y difundirla a través de distintas modalidades simbólicas y mediante diversas fuentes y recursos digitales" (Area et al., 2008: 75). Ello implica tener la capacidad para buscar, analizar y evaluar información específica que circula en la red con el fin de manipularla para llegar a transformarla en conocimiento y emplearla para resolver problemas (Adell, 2010).

La dimensión denominada Ciudadanía digital se vincula con la adopción de una actitud crítica y reflexiva frente a la información que se recibe de los distintos medios de comunicación digital, y a la 
comprensión del impacto social y económico que generan las tecnologías; junto con hacer uso de estas herramientas en forma autónoma y responsable respetando los aspectos legales y defender los propios derechos digitales (Adell, 2010).

Si bien el desarrollo de la competencia digital traspasa todos los ámbitos de especialización, adopta una relevancia crucial en el ámbito de la pedagogía. Por una parte, los procesos formativos de todos los niveles y áreas educativas requieren de la labor que ejerce el profesorado y, por otra, el desarrollo de la competencia digital es una condición necesaria para la formación integral y para el aprendizaje permanente de una persona dentro del contexto actual y, precisamente, dicha responsabilidad recae en la labor que desempeñan los docentes (Flores y Roig, 2016).

Por consiguiente, el profesorado se encuentra con el desafío de incorporar las TIC en su práctica docente para apoyar los procesos de enseñanza y aprendizaje de los estudiantes, pero se espera que lo haga desde una perspectiva pedagógica y no con fines meramente técnicos e instrumentales, como ha ido sucediendo desde hace algún tiempo (Cabero, 2004; Valverde et al., 2010). Al respecto, es necesario destacar que la incorporación de estas herramientas en el proceso de enseñanza y aprendizaje no sólo implica que el docente deba ser capaz de reflexionar en torno al qué, al cómo, al cuándo y para qué utilizarlas, sino que al mismo tiempo se requiere que este actor sea capaz de reflexionar en torno a los modelos epistemológicos bajo los cuales comprende el proceso de enseñanza y aprendizaje, considerando fundamentalmente su pertinencia a la realidad educativa del siglo XXI.

Sumado a lo anterior, no se puede desconocer que en la actualidad, tanto los estudiantes que se encuentran en proceso de formación inicial docente como el profesorado en ejercicio, necesitan tener un profundo conocimiento sobre cómo estas herramientas están a su servicio para favorecer el aprendizaje e innovar en sus prácticas educativas. En este sentido, concordamos con Vaillant (2013: 7), cuando sostiene que "el conocimiento tecnológico es condición necesaria para avanzar en la integración de las TIC, pero no resulta suficiente para innovar. Los docentes requieren hoy conocimientos pedagógicos sobre el uso de las TIC". En consecuencia, las demandas actuales para la formación del profesorado van en la línea de propender al desarrollo de la competencia digital en todas sus dimensiones como cualquier ciudadano, pero al mismo tiempo se busca favorecer el logro de aquellos saberes, procedimientos y actitudes que los habiliten para ejercer una acción didáctica con estas herramientas, articuladas con su ámbito disciplinario. En concreto, los profesores requieren desarrollar además otro tipo de competencia, la competencia didáctica digital.

La competencia didáctica digital puede ser entendida como la capacidad del docente para movilizar sus conocimientos sobre didáctica y articularlos con las funcionalidades de los recursos digitales disponibles, integrándolos en el aula con la finalidad de provocar aprendizajes en los estudiantes, demostrando creatividad y una actitud favorable para aprovechar las potencialidades que estos recursos ofrecen (Flores, 2014). Hace referencia fundamentalmente a un saber práctico del docente, que implica ir más allá de poseer un amplio conocimiento sobre las alternativas digitales disponibles en la red o sobre la existencia de programas educativos específicos para su ámbito disciplinario, pues en rigor requiere saber cómo aplicar dichos conocimientos en su acción pedagógica.

$\mathrm{Si}$ bien en la literatura se pueden encontrar diversas propuestas teóricas que de una u otra forma buscan proporcionar lineamientos respecto a cuáles serían los desempeños deseables para los docentes en el ámbito de las TIC (Cabero y Marín, 2014; Prendes, 2010; Perrenoud, 2004), consideramos pertinente hacer mención a las diversas propuestas de estándares específicos para docentes, porque éstas intentan unificar criterios y proporcionar lineamientos en 
torno a los conocimientos, habilidades y actitudes que deben lograr los docentes para integrar las tecnologías dentro de su práctica pedagógica, de este modo también se transforman en modelos de referencia que orientan y guían el diseño de planes de formación docente para el uso de las TIC en educación (Prendes y Gutiérrez, 2013), pero en el marco de ciertos criterios considerados de calidad y válidos para un determinado contexto.

Entre las alternativas de estándares sobre competencias TIC disponibles específicamente para el profesorado, se encuentran los estándares NETS-T (ISTE, EUA); ICT (Comunidad Europea); Estándares TIC para Formación Inicial Docente (MINEDUC, Chile); Competencias y Estándares TIC para la Profesión Docente (MINEDUC, Chile); RUTA (Colombia); Marco de Competencias TIC para Docentes (UNESCO); Proffesional Standars for Teachers (Australia), 21st Century Knowledge and Skills in Educator Preparation (AACTE, EUA), entre otros. Al analizar específicamente algunos de estos estándares en un trabajo previo (Flores, 2014), se pudo evidenciar la presencia de aspectos comunes en algunos ámbitos, por ejemplo: la definición de objetivos centrados en el mejoramiento de los procesos formativos tanto de los estudiantes como del profesorado - manejo y uso del hardware y software; vinculación de las TIC con el currículum; aspectos éticos y legales.

Dentro de las propuestas mencionadas anteriormente, especial atención merecen los Estándares TIC para la Formación Inicial Docente (FID) (Silva et al., 2008), pues se han focalizado exclusivamente en definir aquellos desempeños que se espera logren los estudiantes de las carreras de Pedagogía al momento de egresar de su proceso formativo. Se estructura en las siguientes cinco dimensiones: Pedagógica; Técnica; Social, ética y legal; Gestión, y Desarrollo profesional, las que al mismo tiempo se articulan con los dos ámbitos básicos de la formación docente, vale decir, formación pedagógica y formación disciplinaria (ver figura 2); integra 16 competencias agrupadas en cada una de las dimensiones señaladas y, al mismo tiempo, ofrece un total de 76 indicadores. Cabe destacar que el aspecto pedagógico es el que presenta un mayor peso, integra siete competencias y 30 indicadores, representando aproximadamente un $44 \%$ del total de competencias definidas (Flores, 2015), y se define de la siguiente forma: "Los futuros docentes adquieren y demuestran formas de aplicar las TIC en el currículo escolar vigente como una forma de apoyar y expandir el aprendizaje y la enseñanza" (Silva et al., 2008: 146).

\section{Metodología}

Para llevar a cabo el estudio se empleó una metodología cuantitativa, específicamente bajo un diseño descriptivo y correlacional, por cuanto ambos permiten estudiar un fenómeno de interés en el medio natural en que se produce sin introducir ninguna manipulación experimental de las variables ni seleccionar los grupos al azar (Castejón, 2006; Cohen y Manion, 2002). En esta investigación sólo nos limitamos a ordenar datos, clasificarlos, medirlos y establecer relaciones no causales entre las variables estudiadas.

El objetivo general del estudio fue determinar aquellos factores que tienen incidencia en la valoración de estudiantes de Pedagogía sobre su nivel de competencia en TIC en la dimensión pedagógica, y para dar respuesta a ello se definieron los siguientes objetivos específicos:

- Establecer la autovaloración que poseen los estudiantes de los niveles formativos de Pedagogía en Educación Parvularia, Educación Básica y Educación Media de la Universidad del BíoBío, sobre el nivel de logro de su competencia en TIC, en la dimensión Aspectos pedagógicos.

- Identificar los factores personales que tienen incidencia en el nivel de competencia digital autopercibido por los estudiantes en la dimensión Aspectos pedagógicos. 


\section{Figura 2. Gráfica dimensiones competencia digital-formación docente}

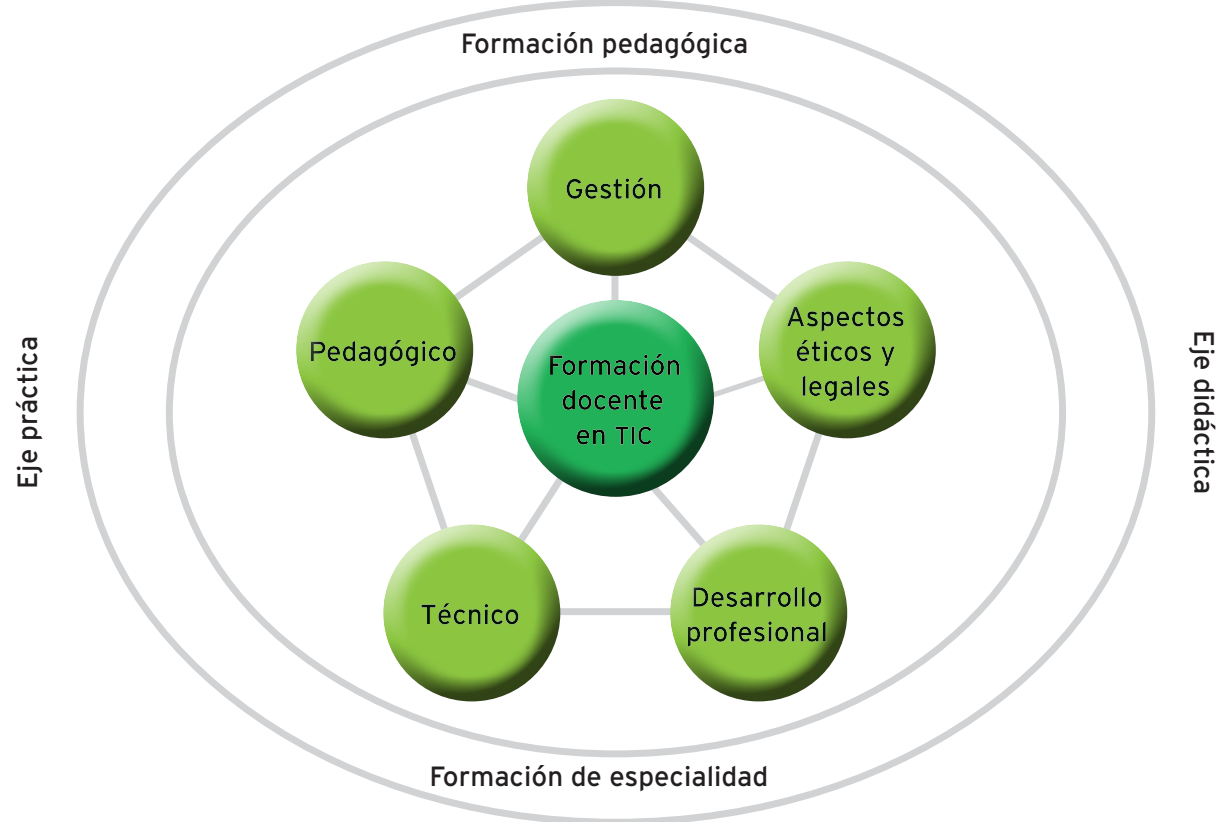

Fuente: Centro de Educación y Tecnología del Ministerio de Educación de Chile [ENLACES] (2008), Estándares en Tecnología de la Información y la Comunicación para la Formación Inicial Docente, p. 145.

\section{Participantes}

La muestra fue de carácter no probabilístico, pues se consideraron aquellos casos que pueden proporcionar el máximo de información (Alaminos, 2006). Particularmente se optó por el muestreo de subgrupos homogéneos, porque los estudiantes que participaron en este estudio comparten una situación común, siendo ésta la realización de la actividad de práctica profesional, ubicada en el último año de formación. La muestra quedó conformada por 175 (n=175) estudiantes de las carreras de Pedagogía de la Facultad de Educación y Humanidades, de la Universidad del Bío-Bío, ubicada en la zona centro sur de Chile. Este valor equivale al 45.8\% del total de estudiantes que asistieron a práctica profesional durante el año académico 2014. El 79.4\% de estudiantes correspondió a mujeres y un $20.6 \%$ a varones. En cuanto a la edad, la media fue de prácticamente 24 años $(\mathrm{SD}=2.51)$.

\section{Variables e instrumento}

Las variables consideradas se organizaron en torno a dos grandes grupos: variables personales y variables evaluativas. Las primeras se diferenciaron a su vez en tres subgrupos: variables sociodemográficas, variables de enseñanza y variables de conectividad. Las segundas hacen referencia a cada una de las dimensiones establecidas en los Estándares TIC para la Formación Inicial Docente (FID) en Chile, vale decir, dimensión Pedagógica; dimensión Aspectos Sociales, Éticos y Legales; dimensión Técnica; Gestión Escolar, y Desarrollo Profesional. Cabe destacar que para este trabajo se consideró específicamente la dimensión Aspectos pedagógicos.

Para la medición de las variables se empleó una escala diseñada ad hoc a la investigación, denominada "Escala de autoevaluación sobre competencia digital para estudiantes de Pedagogía”. Esta escala contiene inicialmente preguntas sobre aspectos sociodemográficos y 
posteriormente 75 reactivos, los que en su globalidad permiten medir cada una de los aspectos que contemplan los Estándares TIC para la FID en el contexto chileno (Silva et al., 2008). Este instrumento fue sometido a un riguroso proceso de validación, tanto de contenido como de constructo (Flores y Roig, 2016) y los resultados obtenidos permitieron determinar la posibilidad de considerar cada una de las dimensiones como una subescala o escala independiente.

Específicamente, la subescala que permitió medir las variables de la dimensión Aspectos pedagógicos contenía 28 indicadores con categorías de respuesta en formato Likert (1: Nunca/Nada; 2: Rara vez/ Poco; 3: A veces/Ni mucho ni poco; 4: Frecuentemente/Bastante, y 5: Mucho/Siempre). Las propiedades psicométricas de este instrumento son bastante satisfactorias, pues el análisis factorial determinó la existencia de seis componentes (ver tabla 1), cuya fiabilidad fue bastante elevada (Alfa de Cronbach = 0.951, IC al 95\%: 0.939 - 0.961) y altamente significativa con $\mathrm{p}<0.001$ (Flores, 2015).

\section{Procedimiento de recogida y análisis de datos}

Una vez que la escala fue diseñada y sometida a un riguroso proceso de validación (Flores y Roig, 2016), se aplicó en forma directa a la muestra considerada en el estudio. Posteriormente se generó una base de datos en el software IBM SSPS Statistics 20, para seguidamente realizar los análisis respectivos.

El tratamiento de la información se llevó a cabo a través del análisis multivariante, entendido éste en un sentido amplio, vale decir, como "todos los métodos estadísticos que analizan simultáneamente medidas múltiples de cada individuo u objeto sometido a investigación. Cualquier análisis simultáneo de más de dos variables puede ser considerado aproximadamente como un análisis multivariante" (Hair et al., 2008: 4). Las técnicas estadísticas utilizadas han dependido principalmente de la naturaleza de cada una de las variables del estudio y se han utilizado las siguientes: análisis descriptivo, Test de diferencias entre medias: $\mathrm{T}$ de Student y su alternativa no-paramétrica el Test U de Mann-Whitney; ANOVA de 1 factor con test de Levene previo y Test de Tukey post-hoc, y su alternativa no paramétrica el Test H de Kruskal-Wallis.

\section{Resultados}

En este apartado se presentan los principales resultados obtenidos a partir de la aplicación de la Escala de autoevaluación de competencia digital para estudiantes de Pedagogía, específicamente en lo referido a la dimensión Aspectos pedagógicos.

En primer lugar, se muestra la información de carácter descriptiva obtenida en cada uno de los factores comprendidos en la escala empleada. Posteriormente, se muestran los resultados respecto al nivel de competencia digital autopercibido por los futuros maestros y finalmente se exhiben los resultados que dan cuenta de aquellas variables que tienen incidencia en el nivel de competencia en el uso de TIC desde la dimensión Aspectos pedagógicos.

\section{Resultados descriptivos sobre el nivel de competencia digital autopercibido por estudiantes de formación inicial en la dimensión "Aspectos pedagógicos"}

En la tabla 2 se presentan los resultados descriptivos obtenidos en cada uno de los seis factores contenidos en la dimensión Aspectos pedagógicos, más la puntuación total de esta dimensión. Se puede observar que los índices de asimetría y curtosis son tolerables (ver tabla 3), sin embargo en cuatro variables el desajuste con respecto al modelo de la curva normal es significativo para $p<0.01$, mientras que en las otras tres variables se podría admitir que se asemejan a una campana de Gauss.

A pesar de la falta de normalidad en algunas de las variables (factores), las medias y medianas respectivas son bastante similares entre sí. Estos estadísticos de centralidad indican que en todos los casos la situación de los estudiantes es bastante semejante y cercana al 


\section{Tabla 1. Factores y reactivos del instrumento de autoevaluación de la dimensión Aspectos Pedagógicos}

\begin{tabular}{|c|c|}
\hline Factores & Ítems \\
\hline \multirow{13}{*}{$\begin{array}{l}\text { 1. Prácticas evaluativas } \\
\text { con TIC y generación } \\
\text { de entornos educativos } \\
\text { virtuales }\end{array}$} & $\begin{array}{l}\text { DPED 23. Aplico estrategias e instrumentos de evaluación soportados por TIC para valorar el } \\
\text { desempeño de los/as estudiantes. }\end{array}$ \\
\hline & $\begin{array}{l}\text { DPED 21. Aplico instrumentos de evaluación para analizar el resultado de mis prácticas docentes con } \\
\text { TIC. }\end{array}$ \\
\hline & $\begin{array}{l}\text { DPED 22. Analizo reflexivamente los resultados y logros alcanzados en experiencias de aprendizaje } \\
\text { desarrolladas con TIC para tomar nuevas decisiones. }\end{array}$ \\
\hline & $\begin{array}{l}\text { DPED 18. Comparo herramientas tecnológicas para seleccionar aquellas que ayudan a atender las } \\
\text { necesidades educativas de los/las estudiantes. }\end{array}$ \\
\hline & $\begin{array}{l}\text { DPED 24. Utilizo los recursos tecnológicos para recolectar y analizar datos, interpretar resultados y } \\
\text { comunicarlos, con el fin de mejorar los aprendizajes de los/as estudiantes. }\end{array}$ \\
\hline & $\begin{array}{l}\text { DPED 25. Favorezco la interacción y el trabajo colaborativo en red a través de estrategias como: } \\
\text { resolución de problemas, método de proyecto, aprendizaje colaborativo, entre otras. }\end{array}$ \\
\hline & $\begin{array}{l}\text { DPED 28. Aplico instrumentos de evaluación que me permiten monitorear el trabajo online en los } \\
\text { procesos de aprendizaje de los/as estudiantes. }\end{array}$ \\
\hline & $\begin{array}{l}\text { DPED 27. Favorezco la participación de todos los/as estudiantes en los entornos virtuales de } \\
\text { aprendizaje que organizo. }\end{array}$ \\
\hline & $\begin{array}{l}\text { DPED 20. Utilizo instrumentos de evaluación para monitorear el aprendizaje de los estudiantes en } \\
\text { entornos de trabajo con TIC. }\end{array}$ \\
\hline & DPED 26. Soy capaz de animar y moderar entornos virtuales de aprendizaje. \\
\hline & $\begin{array}{l}\text { DPED 17. Utilizo criterios (usabilidad, interfaz, accesibilidad e interactividad) para seleccionar software } \\
\text { y recursos tecnológicos posibles para ser usados en mi sector curricular. }\end{array}$ \\
\hline & $\begin{array}{l}\text { DPED 19. Examino innovaciones tecnológicas aplicadas en educación (software educativo, sitios web, } \\
\text { etc.) y recursos digitales existentes en el sistema escolar e internet, para seleccionar aquellos que son } \\
\text { relevantes en mi sector curricular y nivel educativo. }\end{array}$ \\
\hline & $\begin{array}{l}\text { DPED 16. Utilizo diferentes propuestas metodológicas apoyadas en las TIC, como webquest, multimedia } \\
\text { project (MMP), mapas conceptuales, entre otros, para favorecer experiencias de aprendizaje } \\
\text { innovadoras. }\end{array}$ \\
\hline \multirow{6}{*}{$\begin{array}{l}\text { 2. Implementación de TIC } \\
\text { como recurso de apoyo } \\
\text { al aprendizaje }\end{array}$} & $\begin{array}{l}\text { DPED 12. Aplico la tecnología para favorecer la creatividad de los estudiantes y mejorar sus } \\
\text { habilidades. }\end{array}$ \\
\hline & $\begin{array}{l}\text { DPED 14. Utilizo la tecnología para apoyar estrategias didácticas que den respuesta a las diversas } \\
\text { necesidades de los estudiantes. }\end{array}$ \\
\hline & $\begin{array}{l}\text { DPED 15. Aplico actividades en las que incorporo recursos TIC como herramientas de apoyo para } \\
\text { favorecer aprendizajes de mi sector curricular. }\end{array}$ \\
\hline & $\begin{array}{l}\text { DPED 13. Oriento a los estudiantes durante el desarrollo de experiencias de aprendizaje mediadas por } \\
\text { las tecnologías. }\end{array}$ \\
\hline & $\begin{array}{l}\text { DPED 3. Identifico experiencias de aprendizaje de mi sector curricular que utilicen la tecnología, } \\
\text { reconociendo sus fortalezas y desafíos. }\end{array}$ \\
\hline & $\begin{array}{l}\text { DPED 1. Identifico los aprendizajes esperados posibles de desarrollar con la incorporación de las TIC, } \\
\text { dentro del programa pedagógico correspondiente al sector curricular de mi especialidad. }\end{array}$ \\
\hline
\end{tabular}




\begin{tabular}{|c|c|}
\hline \multirow{3}{*}{$\begin{array}{l}\text { 3. Diseño de experiencias } \\
\text { de aprendizaje con TIC }\end{array}$} & DPED 5. Utilizo algunas estrategias de aprendizaje que requieren del uso de Internet. \\
\hline & $\begin{array}{l}\text { DPED 6. Defino entornos de trabajo donde los/las estudiantes necesitan utilizar Internet como medio } \\
\text { para abordar los contenidos seleccionados. }\end{array}$ \\
\hline & $\begin{array}{l}\text { DPED 7. Selecciono estrategias de aprendizaje que demandan el uso de herramientas de productividad } \\
\text { (procesador de texto, planillas de cálculo, software de presentación u otros). }\end{array}$ \\
\hline \multirow{2}{*}{$\begin{array}{l}\text { 4. Preparación de } \\
\text { recursos educativos } \\
\text { digitales. }\end{array}$} & $\begin{array}{l}\text { DPED 10. Empleo herramientas computacionales para el desarrollo de recursos multimediales como } \\
\text { medio de apoyo a las actividades pedagógicas (creación de páginas web y/o aplicaciones para el } \\
\text { desarrollo de éstas). }\end{array}$ \\
\hline & $\begin{array}{l}\text { DPED 11. Diseño objetos de aprendizaje con elementos multimedia para incorporarlos en espacios } \\
\text { virtuales de aprendizaje. }\end{array}$ \\
\hline \multirow{2}{*}{$\begin{array}{l}\text { 5. Conocimiento sobre } \\
\text { posibilidades del uso } \\
\text { de TIC en su ámbito } \\
\text { curricular }\end{array}$} & $\begin{array}{l}\text { DPED 2. Identifico las características y finalidad que poseen algunas herramientas digitales didácticas: } \\
\text { webquest, wiki, web didáctica, blog u otras. }\end{array}$ \\
\hline & $\begin{array}{l}\text { DPED 4. Identifico fuentes impresas y/o digitales que contienen información sobre experiencias } \\
\text { educativas que hacen uso de las TIC en mi especialidad. }\end{array}$ \\
\hline \multirow{2}{*}{$\begin{array}{l}\text { 6. Preparación de } \\
\text { material didáctico con } \\
\text { apoyo de TIC }\end{array}$} & $\begin{array}{l}\text { DPED 9. Utilizo la plantilla de cálculo en la preparación de materiales didácticos de apoyo a los } \\
\text { procesos de enseñanza y aprendizaje, de acuerdo con mi sector curricular y al nivel donde me } \\
\text { desempeño. }\end{array}$ \\
\hline & $\begin{array}{l}\text { DPED 8. Empleo procesadores de texto para producir material didáctico de apoyo a mis actividades } \\
\text { pedagógicas (guías, pruebas, materiales de lectura, entre otros). }\end{array}$ \\
\hline
\end{tabular}

Fuente: Elaboración propia.

Tabla 2. Análisis descriptivo. Variables dimensión Aspectos pedagógicos

\begin{tabular}{|l|c|c|c|c|c|c|c|}
\hline \multicolumn{1}{|c|}{ Factores } & Factor 1 & Factor 2 & Factor 3 & Factor 4 & Factor 5 & Factor 6 & Punt. Total \\
\hline Media & 3.06 & 3.58 & 3.47 & 3.08 & 3.25 & 3.68 & 3.28 \\
\hline IC 95\%: límite inferior & 2.95 & 3.48 & 3.35 & 2.93 & 3.13 & 3.56 & 3.18 \\
\hline IC 95\%: límite superior & 3.18 & 3.68 & 3.58 & 3.23 & 3.37 & 3.79 & 3.37 \\
\hline Error típico de la media & 0.06 & 0.05 & 0.06 & 0.08 & 0.06 & 0.06 & 0.05 \\
\hline Mediana & 3.15 & 3.67 & 3.67 & 3.00 & 3.50 & 3.50 & 3.29 \\
\hline Mínimo & 1.08 & 2.00 & 1.00 & 1.00 & 1.00 & 1.50 & 1.79 \\
\hline Máximo & 4.69 & 5.00 & 5.00 & 5.00 & 5.00 & 5.00 & 4.75 \\
\hline Desviación estándar & 0.78 & 0.68 & 0.79 & 1.01 & 0.82 & 0.79 & 0.64 \\
\hline Varianza & 0.61 & 0.46 & 0.62 & 1.02 & 0.67 & 0.62 & 0.41 \\
\hline Rango semi-intercuartil & 0.58 & 0.42 & 0.50 & 0.75 & 0.75 & 0.50 & 0.45 \\
\hline Coeficiente de Variación & 25.49 & 18.99 & 22.77 & 32.79 & 25.23 & 21.47 & 19.51 \\
\hline
\end{tabular}

Fuente: elaboración propia. 
Tabla 3. Análisis exploratorio. Variables de la dimensión Aspectos pedagógicos. Ajuste al modelo normal

\begin{tabular}{|l|c|c|c|c|c|c|c|}
\hline \multicolumn{1}{|c|}{ Factores } & Factor 1 & Factor 2 & Factor 3 & Factor 4 & Factor 5 & Factor 6 & Punt. Total \\
\hline Asimetría & -0.345 & -0.030 & -0.291 & -0.191 & -0.235 & -0.415 & -0.204 \\
\hline Curtosis & -0.330 & -0.601 & -0.517 & -0.713 & -0.312 & -0.245 & -0.400 \\
\hline TEST K-S: P-SIG & 0.214 NS & 0.138 NS & $0.004 * *$ & $0.002 * *$ & $0.001^{* *}$ & $0.001^{* *}$ & 0.865 NS \\
\hline
\end{tabular}

NS = no significativo $(p>0.050)$ Buen ajuste ** Altamente Significativo al 1\% desvío grave. Fuente: elaboración propia.

punto medio de la escala. Dentro de esta similitud, en el factor 1 y en el 4 es donde se aprecian los valores más bajos mientras que en el 2 y en el 3 los más altos. La variabilidad es pareja entre todas las variables, la mayor homogeneidad se encuentra en la puntuación total y en el factor 3, y la mayor dispersión en el 4.

\section{Incidencia de variables personales sobre el nivel de competencia digital autopercibido por estudiantes de formación inicial en la dimensión pedagógica}

Las variables personales que se consideraron para determinar si tienen incidencia en el nivel de competencia digital autopercibido por los estudiantes de formación inicial docente fueron las siguientes: edad, sexo, nivel de formación inicial docente (Educación parvularia, Educación general básica y Educación media); formación y/o capacitación en TIC; conectividad (frecuencia de acceso a internet, finalidad de navegar por la red, uso de redes sociales).

\section{Estudio del efecto variable edad}

La edad, como se puede apreciar en la figura 3, presenta una clara asimetría hacia la izquierda, es decir con mayor presencia de sujetos de menor edad dentro del rango observado (entre 21 y 36 años), con mediana 23 y media 24 años. Por ello se toma la decisión de categorizarla, tratando de buscar grupos con cierta homogeneidad en su tamaño. Se opta por formar 3 grupos: 21-22 años, donde se incluyen 50 casos (28.6\%); 23-24 años donde quedan comprendidos 69 sujetos (39.4\%) y el tercer grupo de $25-36$ años y más, donde se encuentran los restante 56 participantes $(32 \%)$.

Los resultados de los test estadísticos empleados para el análisis del efecto de la edad sobre el nivel de competencia digital autopercibido por los estudiantes en la dimensión pedagógica se resumen en la tabla 4. Se observan diferencias altamente significativas para $p<0.01$ según ambos test estadísticos $\left[F(2,172)=5.05, p=0.007 ; \chi^{2}(2, \mathcal{N}=172)=10.32\right.$, $p=0.006]$, aunque con un tamaño del efecto pequeño $\left(R^{2}=0.055\right)$. Los test de pares a posteriori agrupan los sujetos de más de 25 años $(M=3.37, S D=0.70)$ con los de 23-24 años $(M=3.38, S D=0.60)$ puesto que no tienen diferencia significativa entre sí $(p>0.05)$, a la vez que ambos grupos tienen medias significativamente mayores $(p<0.05)$ que los sujetos de 21-22 años $(M=3.04, S D=0.58)$. Por consiguiente, se han encontrado algunas diferencias significativas que indican que los sujetos más jóvenes (21-22 años) tienen puntuaciones menores que el resto de casos, fundamentalmente a partir de los 23 años.

\section{Estudio del efecto variable: género}

En la tabla 5 se presentan los resultados obtenidos respecto a la variable género y su incidencia en el nivel de competencia digital autopercibido por los estudiantes en la dimensión aspectos pedagógicos. 
Figura 3. Distribución de la edad en la muestra $(n=171)$

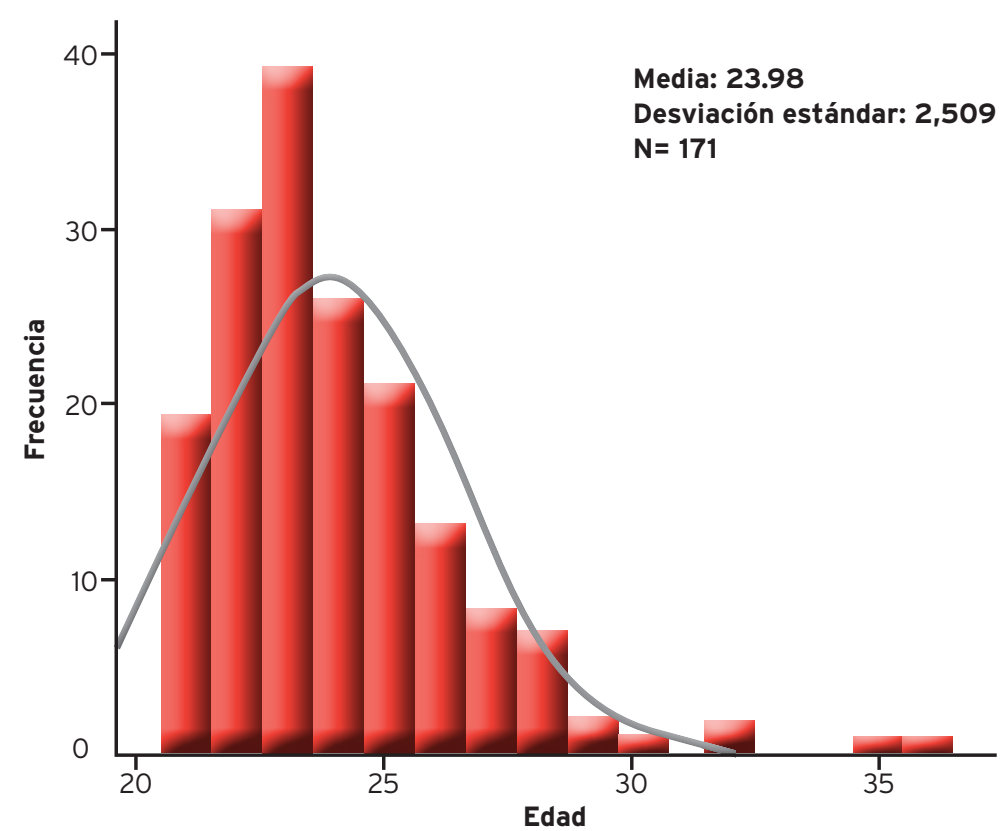

Fuente: elaboración propia.

Tabla 4. Test de diferencia de medias: ANOVA. Puntuaciones de dimensión pedagógica en función de la edad

\begin{tabular}{|c|c|c|c|c|c|c|c|c|c|}
\hline \multirow{2}{*}{$\begin{array}{l}\text { Variable } \\
\text { / edad }\end{array}$} & \multirow{2}{*}{$N$} & \multirow{2}{*}{ Media (IC 95\%) } & \multirow{2}{*}{ D.E. } & \multicolumn{3}{|c|}{ ANOVA 1 factor } & \multirow{2}{*}{ Test KW: } & \multirow{2}{*}{ R2 } & \multirow{2}{*}{$\begin{array}{l}\text { POST-HOC: Tukey } \\
\text { Subconjuntos } \\
\text { homogéneos }\end{array}$} \\
\hline & & & & $\boldsymbol{F}$ & gl & $P$ & & & \\
\hline $21-22 a$ & 50 & $3.04(2.87-3.20)$ & 0.58 & \multirow{3}{*}{5.05} & \multirow{3}{*}{$2 ; 172$} & \multirow{3}{*}{$0.007 * *$} & \multirow{3}{*}{$\begin{aligned} \mathrm{Chi}^{2} & =10.322 \mathrm{gl} \\
\mathrm{P} & =0.006^{* *}\end{aligned}$} & \multirow{3}{*}{0.055} & \multirow{3}{*}{$21-22<[(23-24)=(25-36)]$} \\
\hline $23-24 a$ & 69 & $3.38(3.23-3.52)$ & 0.60 & & & & & & \\
\hline $25-36 a$ & 56 & $3.37(3.18-3.56)$ & 0.70 & & & & & & \\
\hline
\end{tabular}

NS $=$ no significativo $(p>0.050) *$ Significativo al 5\% (p<0.050) ** Altamente significativo al 1\% (p<0.010).

Fuente. Elaboración propia. 
Como se puede apreciar, se han encontrado diferencias $(p<0.05)$ en tres factores.

En el factor 1, la media de los hombres $(M=3.24$, $S D=0.59)$ ha resultado ser más alta que la de las mujeres $(M=2.97, S D=0.80)$; diferencia que es significativa para $\mathrm{p}<0.01$ en ambos test estadísticos $[\mathrm{t}(173)=3.18, \mathrm{p}=0.002 ; z=2.95, p=0.003]$, pero con un tamaño del efecto pequeño $\left(R^{2}=0.055\right)$.

En el factor 5, nuevamente la media de los hombres $(M=3.41, S D=0.73)$ es más alta que la de las mujeres $(M=3.18, S D=0.83)$, es significativo sólo para $p<0.05$ y por ello un tamaño del efecto muy leve $\left(R^{2}=0.027\right)$.

En la puntuación total, también se observa que los hombres obtienen una media $(M=3.53$, $S D=0.50)$ algo más alta que la de las mujeres $(M=3.21, S D=0.66)$, con diferencias estadísticamente significativas para $p<0.01$ entre ambos grupos $[t(173)=2.70, \mathrm{p}=0.008 ; z=2.57, p=0.009]$ con un $\mathrm{IC}$ al 95\%: 0.09-0.55, aunque con un tamaño del efecto que sigue siendo pequeño $\left(R^{2}=0.040\right)$.

Tabla 5. Test de diferencia de medias. Factores y puntuación total dimensión aspectos pedagógicos según variable sexo

\begin{tabular}{|c|c|c|c|c|c|c|c|c|c|c|c|}
\hline \multirow{2}{*}{\multicolumn{2}{|c|}{$\begin{array}{c}\text { Variable/ } \\
\text { Grupo }\end{array}$}} & \multirow{3}{*}{$\begin{array}{l}N \\
36\end{array}$} & \multirow{3}{*}{$\begin{array}{l}\text { Media (IC 95\%) } \\
3.42(3.22-3.62)\end{array}$} & \multirow{3}{*}{$\begin{array}{l}\text { D.E. } \\
0.59\end{array}$} & \multicolumn{3}{|c|}{$T$ Student } & \multicolumn{2}{|c|}{ Test MW } & \multirow{2}{*}{ IC 95\% dif } & \multirow{2}{*}{$\begin{array}{l}\text { Tamaño del } \\
\text { efecto: } R^{2}\end{array}$} \\
\hline & & & & & $T$ & $a !$ & $P$ & 7 & 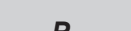 & & \\
\hline & Hombre & & & & \multirow{2}{*}{3.18} & \multirow{2}{*}{173} & \multirow{2}{*}{$0.002 * *$} & \multirow{2}{*}{2.95} & \multirow{2}{*}{$0.003 * *$} & \multirow{2}{*}{$0.17-0.73$} & \multirow{2}{*}{0.055} \\
\hline & Mujer & 139 & $2.97(2.84-3.10)$ & 0.80 & & & & & & & \\
\hline \multirow{2}{*}{ Fac.2 } & Hombre & 36 & $3.73(3.51-3.95)$ & 0.65 & \multirow{2}{*}{1.55} & \multirow{2}{*}{173} & \multirow{2}{*}{0.124 NS } & \multirow{2}{*}{1.55} & \multirow{2}{*}{$0.120 \mathrm{NS}$} & \multirow{2}{*}{--} & \multirow{2}{*}{0.014} \\
\hline & Mujer & 139 & $3.54(3.42-3.65)$ & 0.68 & & & & & & & \\
\hline \multirow{2}{*}{ Fac.3 } & Hombre & 36 & $3.59(3.38-3.81)$ & 0.64 & \multirow{2}{*}{1.07} & \multirow{2}{*}{173} & \multirow{2}{*}{0.186 NS } & \multirow{2}{*}{0.94} & \multirow{2}{*}{0.349 NS } & \multirow{2}{*}{---} & \multirow{2}{*}{0.007} \\
\hline & Mujer & 139 & $3.44(3.30-3.57)$ & 0.82 & & & & & & & \\
\hline \multirow{2}{*}{ Fac.4 } & Hombre & 36 & $3.31(2.98-3.63)$ & 0.97 & \multirow{2}{*}{1.51} & \multirow{2}{*}{173} & \multirow{2}{*}{0.134 NS } & & & & \\
\hline & Mujer & 139 & $3.02(2.85-3.19)$ & 1.02 & & & & & & & \\
\hline & Hombre & 36 & $3.51(3.27-3.76)$ & 0.73 & & & & & & & \\
\hline rac.s & Mujer & 139 & $3.18(3.04-3.32)$ & 0.83 & 2.18 & $1 / 3$ & $0.031^{*}$ & 2.03 & $0.042^{*}$ & $0.03-0.63$ & 0.027 \\
\hline & Hombre & 36 & $3.78(3.52-4.04)$ & 0.77 & & & & & & & \\
\hline & Mujer & 139 & $3.65(3.52-3.78)$ & 0.79 & & & & & & & \\
\hline & Hombre & 36 & $3.53(3.36-3.70)$ & 0.50 & & & & & & & \\
\hline r. 100. & Mujer & 139 & $3.21(3.19-3.32)$ & 0.66 & 2.10 & & 0.008 & 2.51 & $0.009^{\circ}$ & $0.09-0.55$ & 0.040 \\
\hline
\end{tabular}

NS $=$ no significativo $(p>0.050) *$ Significativo al $5 \%(p<0.050) * *$ Altamente significativo al $1 \%(p<0.010)$ Fuente. Elaboración propia. 


\section{Estudio del efecto variable: nivel educativo de formación inicial}

Una vez que se ha hecho la exploración de los datos, se procede a reagrupar las diferentes carreras de los estudiantes participantes en los tres niveles de formación inicial docente existentes en Chile, vale decir, Educación Parvularia, Educación Básica y Educación Media. Tras esta agrupación la muestra queda conformada por 41 alumnas de Educación parvularia (23.4\%), 61 estudiantes de Básica (34.9\%) y 73 estudiantes de Media (41.7\%) (ver figura 4).

A continuación se compara a estos tres grupos en cuanto a las variables definidas como puntuación total de la dimensión Aspectos pedagógicos. Para el contraste comparativo se utilizó la técnica paramétrica Análisis de Varianza (ANOVA) de 1 factor de efectos fijos, comprobando la condición previa de homocedasticidad (Test de Levene), estimando el tamaño del efecto con $R^{2}$ y acudiendo a los contrastes por pares post-hoc (Test de Tukey) cuando se encuentre significación. La falta de normalidad en algunas de estas variables recomienda corroborar los resultados de ANOVA con su alternativa no paramétrica, el Test $\mathrm{H}$ de Kruskal-Wallis (KW), que en cualquier caso ayuda a aumentar la confiabilidad estadística.

La condición de homocedasticidad entre grupos se ha cumplido en todas las variables $(p>0.05$ en los respectivos Test de Levene), por tanto queda legitimado el uso de la técnica de Análisis de Varianza. Los resultados de estos ANOVA y sus test alternativos de KW han sido muy similares, como era de esperar, salvo en una variable donde hay una pequeña variación. El resumen de estos resultados se presenta en la tabla 6 y posteriormente se comentan cada una de las variables.

Como se puede observar, se han encontrado diferencias altamente significativas para $p<0.01$ confirmadas por ambos test estadísticos $[F(2,172)=5.64$, $\left.p=0.004 ; \chi^{2}(2, \mathrm{~N}=172)=14,22, p=0.001\right] . \mathrm{El}$ tamaño del efecto es pequeño $\left(\mathrm{R}^{2}=0.62\right)$. Los test de pares post-hoc, agrupan los niveles de Educación básica

\section{Figura 4. Distribución de la muestra de participantes según su nivel de formación inicial docente}

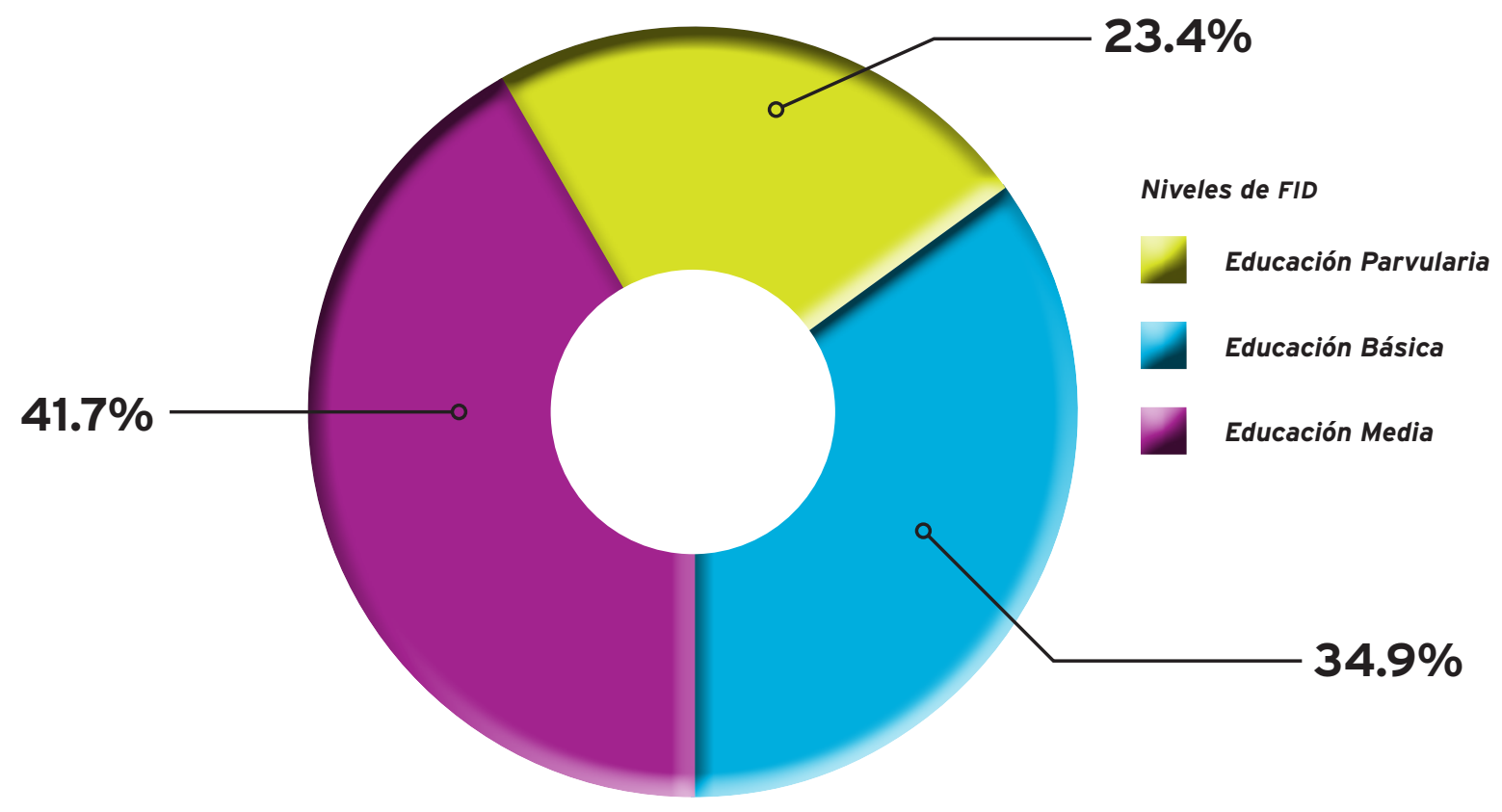


Tabla 6. Efecto de variable nivel educativo de formación inicial en el nivel de competencia digital autopercibido en la dimensión aspectos pedagógicos

\begin{tabular}{|c|c|c|c|c|c|c|c|c|c|}
\hline \multirow{2}{*}{$\begin{array}{c}\text { Variable/ } \\
\text { FID }\end{array}$} & \multirow{2}{*}{$N$} & \multirow{2}{*}{ Media (IC 95\%) } & \multirow{2}{*}{ D.E. } & \multicolumn{3}{|c|}{ ANOVA 1 factor } & \multirow{2}{*}{ Test KW: } & \multirow{2}{*}{$R^{2}$} & \multirow{2}{*}{$\begin{array}{l}\text { POST-H } \\
\text { OC: Tukey } \\
\text { Subconju. } \\
\text { homogén. }\end{array}$} \\
\hline & & & & $\boldsymbol{F}$ & gl & $P$ & & & \\
\hline Ed. Parv. (P) & 41 & $2.99(2.77-3.22)$ & 0.72 & \multirow{3}{*}{5.64} & \multirow{3}{*}{$2 ; 172$} & \multirow{3}{*}{$0.004 * *$} & \multirow{3}{*}{$\begin{array}{r}W x^{2}=14.22 \\
2 g l \\
P=0.001^{* *}\end{array}$} & \multirow{3}{*}{0.062} & \multirow{3}{*}{$P<(B=M)$} \\
\hline Ed. Bas. (B) & 61 & $3.39(3.23-3.56)$ & 0.64 & & & & & & \\
\hline Ed. Med.(M) & 73 & $3.34(3.21-3.47)$ & 0.56 & & & & & & \\
\hline
\end{tabular}

NS $=$ No significativo $(p>0.05) *$ Significativo al $5 \% * *$ Altamente Significativo al $1 \%$ Fuente. Elaboración propia.

$(M=3.39, S D=0.64)$ y Media $(M=3.34, S D=0.56)$, que presentan diferencias entre sí $(p>0.05)$, y que tienen un valor medio significativamente superior $(p<0.01)$ que las estudiantes del nivel de Parvularia $(M=2.99, S D=0.72)$.

\section{Estudio del efecto variable: tipo de capacitación en TIC}

A continuación se presentan los resultados obtenidos en la evaluación del posible efecto de haber recibido alguna capacitación en TIC sobre la competencia digital autopercibida en la dimensión aspectos pedagógicos. Los resultados se resumen en la tabla 7, donde se puede apreciar la existencia de una diferencia significativa para $p<0.01$ en ambos test estadísticos empleados $[t(171)=2.84, p=0.005 ; Z=2.77, p=0.006]$, según la cual la media de los estudiantes que tienen capacitación $(M=3.47, S D=0.57)$ es más alta que la de los que no la tienen $(M=3.18, S D=0.65)$, con tamaño del efecto pequeño $\left(R^{2}=.045\right)$.

\section{Estudio del efecto variable: conectividad a internet}

En cuanto a la posible relación entre la disponibilidad de acceso a internet en su móvil y el nivel de competencia digital autopercibido por los estudiantes en la dimensión aspectos pedagógicos, se observa que no hay ningún tipo de relación, ya que las diferencias observadas no alcanzan significación $(p>0.05)$ (ver tabla 8).

\section{Tabla 7. Test de diferencia de medias. Puntuaciones de dimensión aspectos pedagógicos en función de su capacitación en TIC}

\begin{tabular}{|c|c|c|c|c|c|c|c|c|c|c|}
\hline \multirow{2}{*}{ Capacitación } & \multirow{2}{*}{$N$} & \multirow{2}{*}{ Media (IC 95\%) } & \multirow{2}{*}{ D.E. } & \multicolumn{3}{|c|}{$T$ Student } & \multicolumn{2}{|c|}{ Test MW } & \multirow{2}{*}{ IC $95 \%$ dif } & \multirow{2}{*}{$\begin{array}{l}\text { Tamaño del } \\
\text { efecto: } R^{2}\end{array}$} \\
\hline & & & & $T$ & gl & $P$ & $z$ & $P$ & & \\
\hline Si & 54 & $3.47(3.32-3.63)$ & 0.57 & \multirow{2}{*}{2.84} & \multirow{2}{*}{171} & \multirow{2}{*}{$0.005^{* *}$} & \multirow{2}{*}{2.77} & \multirow{2}{*}{$0.006 * *$} & \multirow{2}{*}{$0.09-0.50$} & \multirow{2}{*}{0.045} \\
\hline No & 119 & $3.18(3.06-3.30)$ & 0.65 & & & & & & & \\
\hline
\end{tabular}

NS $=$ no significativo $(p>0.050) *$ Significativo al $5 \%(p<0.050) * *$ Altamente significativo al $1 \% p<0.010)$ Fuente. Elaboración propia. 
Tabla 8. Test de diferencia de medias. Puntuaciones de competencia digital en la dimensión pedagógica, en función de tener conexión a internet en el móvil

\begin{tabular}{|c|c|c|c|c|c|c|c|c|c|c|}
\hline \multirow{2}{*}{$\begin{array}{l}\text { Internet en } \\
\text { móvil }\end{array}$} & \multirow{2}{*}{$N$} & \multirow{2}{*}{ Media (IC 95\%) } & \multirow{2}{*}{ D.E. } & \multicolumn{3}{|c|}{$T$ Student } & \multicolumn{2}{|c|}{ Test MW } & \multirow{2}{*}{ IC 95\% dif } & \multirow{2}{*}{$\begin{array}{l}\text { Tamaño del } \\
\text { efecto: } R^{2}\end{array}$} \\
\hline & & & & $T$ & gl & $P$ & $z$ & $P$ & & \\
\hline $\mathrm{Si}$ & 130 & $3.30(3.20-3.41)$ & 0.73 & \multirow{2}{*}{0.85} & \multirow{2}{*}{173} & \multirow{2}{*}{0.396 NS } & \multirow{2}{*}{0.91} & \multirow{2}{*}{0.365 NS } & \multirow{2}{*}{--} & \multirow{2}{*}{0.004} \\
\hline No & 45 & $3.21(2.99-3.43)$ & 0.61 & & & & & & & \\
\hline
\end{tabular}

$N S=$ no significativo $(p>0.050) *$ Significativo al $5 \%(p<0.050) * *$ Altamente significativo al $1 \% p<0.010)$

Fuente. Elaboración propia.

\section{Discusión y conclusiones}

No se puede negar que desde hace algún tiempo el profesorado se ha visto enfrentado al desafío de tener que incorporar las TIC dentro de su práctica pedagógica, pues se han instalado una serie de políticas orientadas a promover la implementación de estas herramientas al interior de los centros educativos con el fin de optimizar los procesos administrativos y pedagógicos. Si bien estas políticas buscan precisamente dar respuesta a las demandas actuales en este ámbito, diversos autores evidencian que la incorporación de TIC en el aula no ha generado el impacto esperado en el mejoramiento de los resultados de aprendizaje o ha sido más lento que en otras áreas (González y de Pablos, 2015; Valverde et al., 2010; Vaillant, 2013; Vásquez-Cuperio y LópezPenedo, 2016), por lo que se desprende que el uso pedagógico de estas herramientas en el aula es un reto que persiste y que debe ser asumido desde la formación inicial del profesorado (Hepp, 2012; Gutiérrez et al., 2010; Vaillant, 2013, 2014).

Si bien los resultados descriptivos de este estudio muestran que los futuros docentes consideran poseer ciertos conocimientos para el uso pedagógico de las TIC en su labor docente, creemos que para avanzar hacia la incorporación real y efectiva de estas herramientas en el aula escolar es necesario continuar en esta línea formativa, pero teniendo en consideración que no es suficiente con que un futuro pedagogo o un docente en ejercicio posea un amplio bagaje de conocimientos sobre estas herramientas, porque el desafío está en saber enseñar con ellas. Del mismo modo, a lo largo de todo el proceso de formación de futuros docentes, se requiere observar detenidamente determinados factores que tienen incidencia en el nivel de competencia autopercibido por los estudiantes en lo que respeta al uso pedagógico de TIC, entre ellos, por ejemplo, las variables género, nivel educativo para el cual están siendo formados y los niveles de capacitación en el uso de las TIC.

En cuanto a la variable género, los resultados de esta investigación muestran que los jóvenes se sienten más capaces en el uso pedagógico de las TIC que sus compañeras $[t(173)=2.70, p=0.008]$, fenómeno que también es coincidente con otros estudios que evidencian resultados similares en torno a la medición de esta variable (Almerich et al., 2011; Papanastasiou y Angeli, 2008; Ponce y Castillo, 2010; Suárez-Rodríguez et al., 2012; Verhoeven et al., 2014). Las diferencias encontradas pueden ser explicadas teniendo en consideración determinados imaginarios sociales que vinculan el ámbito tecnológico y las competencias asociadas a su empleo, más bien a un área de dominancia masculina (Cabero, Marín y Vázquez, 2011; Gil-Juárez, Feliú y Vitores, 2012). 
Otro de los factores que tendría incidencia en el nivel de desempeño en el uso pedagógico de estas herramientas, de acuerdo con los resultados de este estudio, corresponde al nivel educativo de formación, pues se evidencia que las estudiantes de Pedagogía en Educación Parvularia sienten poseer un nivel de desempeño más bajo que sus compañeros de las carreras de Pedagogía en Educación Básica y Educación Media. Este resultado es similar al de otros estudios que han considerado la medición de esta variable (Hsu, 2010; Hsu y Kuan, 2013; Valcke, Rots, Verbeke y Van Braak, 2007), donde se evidencia que los profesores con grados avanzados tienen una mayor integración de las TIC en el proceso de enseñanza y aprendizaje.

En relación con lo anterior, consideramos importante enfatizar sobre la necesidad de que en la formación inicial de docentes se tengan en cuenta simultáneamente las variables "género y nivel educativo de formación", por cuanto los resultados resultan ser preocupantes sí se tiene en consideración la condición de feminización de la carrera docente en los niveles de educación infantil y educación primaria o básica (Sáinz, 2013), porque podría suponerse entonces que los niños y niñas que asisten a estos niveles educativos, al estar mayoritariamente bajo la responsabilidad de una profesora, se encuentran frente a una clara desventaja respecto a las formas de interacción con las tecnologías digitales en el aula, al compararlos con los niveles educativos superiores en los que existe mayor presencia de docentes varones.

Por otro lado, es importante también tener en cuenta que para que los futuros docentes se sientan capacitados en el uso de las TIC desde una perspectiva pedagógica, el proceso formativo debe ofrecerles alternativas de formación en esta línea que vayan más allá de cursar sólo una determinada asignatura de tecnología, instalada por lo demás en el primer año de formación, ya que los resultados de este estudio sugieren que los estudiantes que han tenido algún tipo de capacitación en TIC se autoevalúan mejor en la dimensión Aspectos pedagógicos de la competencia digital que aquellos que no la han tenido, lo que además está en la línea de otros estudios que han determinado que el profesorado que ha tenido algún tipo de capacitación tienden a utilizar con mayor frecuencia estas herramientas y lo hacen con mayor seguridad (Pozuelo, 2014).

A partir de la revisión de la literatura disponible respecto al ámbito aquí presentado y de los resultados obtenidos en este estudio, creemos necesario destacar que la formación de futuros docentes en el uso de las TIC, además de estar focalizada hacia el desarrollo de cada una de las dimensiones de la competencia digital y hacia la construcción de saberes prácticos vinculados que le permitan saber enseñar con estas herramientas, debe modelar también ciertos factores personales que favorecen la incorporación de las TIC en la práctica docente, entre ellos, la disposición para apropiarse de estas herramientas, la autonomía en el aprendizaje, la creatividad, la reflexión crítica y la actitud positiva frente a las herramientas y digitales.

Teniendo en consideración los antecedentes teóricos sobre la competencia digital y los resultados obtenidos en este estudio, concluimos que la formación de los futuros docentes en el uso pedagógico de TIC debe visualizarse desde una perspectiva transversal e integradora, focalizada más hacia "saber ser y hacer con las TIC" que al "saber sobre las TIC", porque en la actualidad no es suficiente que el profesorado posea amplios conocimientos sobre las posibilidades educativas que las herramientas tecnológicas y digitales ofrecen, por cuanto lo relevante, a nuestro juicio, se visualiza en la capacidad que tenga el docente para ejercer una acción pedagógica con ellas.

Finalmente, consideramos necesario destacar que para el desarrollo de esta investigación se ha utilizado un diseño metodológico centrado en un estudio de caso y la muestra considerada es bastante reducida, por tanto, los resultados corresponden a una 
realidad específica y no permiten hacer generalizaciones o ser transferibles a otras realidades educativas. Por otra parte, al centrarse exclusivamente en la autoevaluación de los estudiantes sobre su competencia digital, la información que ellos registraron

\section{Referencias}

Adell, J. (2010), "Las 5 dimensiones de la competencia digital", Parte II de la ponencia "La Competencia Digital", XXIV Jornadas Pedagógicas de Barakaldo, $<$ https://www.youtube.com/watch?v=qWLTM r6ZmzM> [Consulta: junio 2016].

Adell, J. (2008), "Competencia digital”, Ponencia presentada en las $2^{\text {a }}$ Jornadas Provinciales Centros TIC, Málaga, 9 y 10 septiembre, <http://www. juntadeandalucia.es / averroes / mochiladigital / didactica/adell.html> [Consulta: mayo 2016].

Alaminos, A. (2006), "La dinámica de la interacción", en A. Alaminos y J.L. Castejón, Elaboración, análisis e interpretación de encuestas, cuestionarios y escalas de opinión, Alcoy, Editorial Marfil, pp. 69-91.

Almerich, G., J. Suárez-Rodríguez, G. Belloch y R. Bo (2011), "Las necesidades formativas del profesorado en TIC: perfiles formativos y elementos de complejidad", en Relieve, vol. 17, núm. 2, pp. 2-28, <http://www. uv.es/RELIEVE/v17n2/RELIEVEv17n2_1.pdf> [Consulta: junio 2014].

Cabero, J. (2004), "Formación del profesorado. El gran caballo de batalla", en Comunicación y Pedagogía. Tecnologías y Recursos didácticos, núm. 195, pp. 27-31, <http://tecnologiaedu.us.es/cuestionario/bibliovir/ jca11.pdf $>$ [Consulta: julio 2016].

Cabero, J. y V. Marín (2014), "Miradas sobre la formación del profesorado en tecnologías de información y comunicación (TIC)", en Enl@ce: Revista Venezolana de Información de Tecnología y Conocimiento, vol. 1, núm. 2, pp. 11-24.

Cabero, J., V. Marín y M. C. Llorente (2012), Desarrollar la competencia digital. Educación mediática a lo largo de toda la podrían tener ciertos sesgos, propios de un proceso subjetivo, lo que en alguna medida podría haber influido en los resultados obtenidos, por lo que sugerimos que se siga indagando en esta línea de investigación.

vida, Sevilla, Editorial MAD.

Cabero, J., V. Marín y A. I. Vázquez (2011), "La mujer y la investigación en tecnología educativa. Análisis de su presencia en la autoría de artículos científicos", en Revista Teoría de la Educación: Educación y Cultura en la Sociedad de la Información, 12(2), pp. 122-148, <http://campus.usal. es/ revistas_trabajo/index.php/revistatesi/article/ view/8276/8280> [Consulta: julio 2016].

Castejón, J. L. (2006), "Escalamiento: medida de las actitudes, opiniones, sentimientos y percepciones", en A. Alaminos y J.L. Castejón, Elaboración, análisis e interpretación de encuestas, cuestionarios y escalas de opinión, España, Alcoy, Editorial Marfil.

Cohen, L. y L. Manion (2002), Métodos en investigación educativa, Madrid, La Muralla.

Comisión de las Comunidades Europeas (2005), "Propuesta de recomendación del Parlamento Europeo y del Consejo sobre las competencias clave para el aprendizaje permanente", Bruselas [on line], <http:// www.europarl.europa.eu/meetdocs/2004_2009/ documents/com/com_com(2005)0548_/com_ com(2005)0548_es.pdf > [Consulta: junio 2015].

Flores, C. (2014), "Competencia digital docente: desempeños didácticos en la formación inicial del profesorado", en Revista científica de educación y comunicación. Hachetetepe, núm. 9, pp. 55-68.

Flores Lueg, C. (2015), "Factores que inciden en la valoración de estudiantes de Pedagogía sobre sus competencias digitales: el caso de la Universidad del Bío-Bío (Chile)", Tesis Doctoral, RUA, Repositorio Institucional Universidad de Alicante, http://hdl. handle.net/10045/50256 [Consulta: junio 2016]. 
Flores-Lueg, C. y R. Roig-Vila, R. (2016), "Diseño y validación de una escala de autoevaluación de competencias digitales para estudiantes de pedagogía", en Pixel-Bit, Revista de Medios y Educación, núm. 48, pp. 209-244, DOI: <http://dx.doi.org/10.12795/ pixelbit.2016.i48.14.> [Consulta: junio 2016].

Fainholc, B., H. Navia, R. Romero y C. Halal (2013), "La formación del profesorado y el uso pedagógico de las TIC", en RED. Revista de Educación a Distancia, núm. 38, pp. 1-14, <http://www.um.es/ead/red/38/ fainholc.pdf $>$ [Consulta: mayo 2015].

Ferrari, A. (2013), DIGCOMP: A framework for developing and understanding digital competence in Europe, Luxemburgo, Publicatioons Office of the European Unión, < http://ftp. jrc.es/EURdoc/JRC83167.pdf> [Consulta: junio 2014].

Gil-Juárez, A., J. Feliú y A. Vitores (2012), "Género y TIC: en torno a la brecha digital de género", en Athenea Digital, vol. 12, núm. 3, pp. 3-9, <http:/ /atheneadigital. net/article/viewFile/v12-n3-gil-feliu-vitores/1137pdf-es> [Consulta: agosto 2016].

Gisbert, M., G. Espuny y J. González (2011), "INCOTIC. Una herramienta para la@utoevaluación diagnóstica de la competencia digital en la Universidad", en Profesorado. Revista de Currículum y Formación del Profesorado, vol. 15, núm. 1, pp. 76-90, <http://digibug.ugr. es/bitstream/10481/15327/1/rev151ART5.pdf> [Consulta: septiembre 2016].

González Pérez, A. y J. de Pablos (2015), "Factores que dificultan la integración de las TIC en las aulas", en Revista de Investigación Educativa, vol. 33, núm. 2, pp. 401-417, DOI: <http://dx.doi.org/10.6018/ rie.33.2.198161> [Consulta: septiembre 2016].

Gutiérrez, A. (2009), "Formación del profesorado y tecnologías de la información y la comunicación. Renovación y convergencia para la educación 2.0 en el "(Ciber) Espacio Europeo de Educación Superior", en Revista Electrónica Teoría de la Educación. Educación y Cultura en la Sociedad de la Información, vol. 10, núm. 1, pp. 93-11, <https://goo.gl/d3sg4R> [Consulta: enero 2015].

Gutiérrez, A., A. Palacios y L. Torrego (2010), "La formación de los futuros maestros y la integración de las
TIC en la educación: anatomía de un desencuentro", en Revista de Educación, núm. 352, <http://www. revistaeducacion.educacion.es/re352/re352_TIC. pdf> [Consulta: marzo 2016].

Gutiérrez, I. (2014), "Perfil del profesor universitario español en torno a las competencias en tecnologías de la información y de la comunicación", en Pixel-Bit. Revista de Medios y Educación, núm. 44, pp. 51-65, DOI: <http://dx.doi.org/10.12795/pixelbit.2014.i44.04> [Consulta: marzo 2015].

Hair, J., R. Anderson y W. Black (2005), Análisis Multivariante, Madrid, Prentice Hall.

Hepp, P. (2012), Caracterización de buenas prácticas con TIC en la formación inicial docente, Buenos Aires, RELPE, <http:// www.relpe.org/wp-content/uploads/2013/04/12Caracterizaci $\% \mathrm{C} 3 \% \mathrm{~B} 3 \mathrm{n}$-de-buenas-pr $\% \mathrm{C} 3 \%$ Alcticasen-formaci $\% \mathrm{C} 3 \% \mathrm{~B} 3 \mathrm{n}$-inicial-docente-en-TIC.pdf> [Consulta: enero 2015].

Hsu, Sh. (2010), "Developing a scale for teacher integration of information and communication technology in grades 1-9", en Fournal of Computer Asssietd Learning, vol. 26, núm. 3, pp. 175-189.

Hsu, Sh. y P. Y. Kuan (2013), “The impact of multilevel factors on technology integration: the case of Taiwanese grade 1-9 teachers and schools", en Education Tech Research Dev, núm. 61, pp. 25-50, DOI: <10.1007/ s1 1423-012-9269-y> [Consulta: enero 2015].

Larrazt, V. (2012), "La competencia digital a la universidad", Tesis doctoral, disponible en TDR (Tesis Doctorales en Red), http://www.tdx.cat/ handle/10803/113431 [Consulta: junio 2016].

Papanastasiou, E. y C. Angeli (2008), "Evaluating the use of ICT in education: psychometric properties of the survey of factors affecting teachers teaching with technology (SFA-T3)", en Educational Technology Ë Society, vol. 11, núm. 1, pp. 69-86.

Perrenoud, P. (2004), Diez nuevas competencias básicas para enseñar, Barcelona, Editorial Grao.

Ponce, R. y N. Castillo (2010), "Una mirada de género al uso de TIC en la educación superior”, en J. Sánchez (ed.), Congreso Iberoamericano de Informática Educativa, 1, 
Santiago de Chile, pp. 763-770.

Pozuelo, J. (2014), “¿Y si enseñamos de otra manera? Competencias digitales para el cambio metodológico", en Caracciolos. Revista digital de investigación en docencia, vol. 2, núm. 1, <http://www3.uah.es/caracciolos/index. $\mathrm{php} /$ caracciolos/article/view/17/27> [Consulta: marzo 2015].

Prendes, M. P. (dir.) (2010), Competencias TIC para la docencia en la universidad pública española. Indicadores y propuestas para la definición de buenas prácticas. Programa de estudio y análisis, <http://www.um.es/competenciastic/informe_final_ competencias2010.pdf> [Consulta: septiembre 2014].

Prendes, M. P. e I. Gutiérrez (2013), "Competencias tecnológicas del profesorado en las universidades españolas", en Revista de Educación, núm. 361, pp. 196222, DOI: <10.4438/1988-592X-RE-2011-361-140> [Consulta: septiembre 2016].

Sáinz, M. (2013), "El uso de las TIC en el ámbito educativo con perspectiva de género. Actitudes del profesorado y del alumnado", en TELOS (Cuadernos de Comunicación e Innovación), núm. I, pp. 1-8, <https:// telos.fundaciontelefonica.com/url-direct/pdf-generato r?tipoContenido $=$ articulo Telos\&idContenido $=20130$ $62110010002 \&$ idioma $=\mathrm{es}>$ [Consulta: marzo 2014].

Silva, J., B. Gros, J. Garrido y J. Rodríguez (2008), "Propuesta de Estándares TIC para la Formación Inicial Docente", en Centro de Educación y Tecnología del Ministerio de Educación de Chile (ENLACES), Estándares TIC para la Formación Inicial Docente. Una propuesta en el contexto chileno, Santiago de Chile, pp. 141173, <http://goo.gl/z3IlTg> [Consulta: mayo 2016].

Suárez-Rodríguez, J., G. Almerich, I. Díaz-García y R. Fernández-Piqueras (2012), “Competencias del profesorado en las TIC. Influencia de factores personales y contextuales", en Universitas Psychologica, vol. 11, núm.
1, pp. 293-309, <http://revistas.javeriana.edu.co/ index.php/revPsycho/article/viewFile/997/1537> [Consulta: julio 2014].

Vaillant, D. (2014). "Formación de profesores en escenarios TIC", en Revista e-Curriculum, vol. 2, núm. 12, pp. 1128-1142, <http://goo.gl/lHYB2y> [Consulta: enero 2015].

Vaillant, D. (2013), "Las políticas de formación docente en América Latina. Avances y desafios pendientes", en M. Poggi (coord.), Politicas docentes: formación, trabajo y desarrollo Profesional, Ciudad Autónoma de Buenos Aires, Instituto Internacional de Planeamiento de la Educación IIPE-Unesco, pp. 45-57, <http://unesdoc. unesco.org/images/0022/002252/225260s.pdf> [Consulta: enero 2015].

Valverde, J., M. Garrido y R. Fernández (2010), "Enseñar y aprender con tecnologías: Un modelo teórico para las buenas prácticas con TIC", en TESI, vol. 11, núm. 3, pp. 203-229, <http://revistatesi.usal. es/ revistas_trabajo/index.php/revistatesi/article/ view/5840/58> [Consulta: octubre 2014].

Vásquez-Cuperio, S. y S. López-Penedo (2016), "Escuela, TIC e innovación educativa", en Digital Education Review, núm. 30, pp. 248-261, <http://revistes.ub.edu/index. $\mathrm{php} / \mathrm{der} / \mathrm{article} / \mathrm{view} / 15367 / \mathrm{pdf}>$ [Consulta:].

Valcke, M., I. Rots, M. Verbeke y J. van Braak (2007), "ICT teacher training: evaluation of the curriculum and training approach in Flanders", en Teaching and Teacher Education: An International Journal of Research and Studies, vol. 23, núm. 6, pp. 795-808.

Verhoeven, J., D. Heerwegh y K. de Wit (2014), "ICT learning experience and research orientation as predictors of ICT skills and the ICT use of university students", en Educ Inf Technol, DOI: <10.1007/s10639014-9310-3> [Consulta: diciembre 2016].

\section{Cómo citar este artículo:}

Flores-Lueg, Carolina y Rosabel Roig-Vila (2019), "Factores personales que inciden en la autovaloración de futuros maestros sobre la dimensión pedagógica del uso de TIC", en Revista Iberoamericana de Educación Superior (RIES), México, UNAM-IISUE/Universia, vol. X, Núm. 27, pp. 151-171, DOI: dx.doi.org/10.22201/ iisue.20072872e.2019.27.345 [consulta: fecha de última consulta]. 